\section{HIGH QUALITY NEOANTIGENS ARE IMMUNOEDITED IN LONG-TERM PANCREATIC CANCER SURVIVORS}

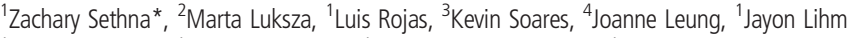
${ }^{1}$ David Hoyos, ${ }^{1}$ Anton Dobrin, ${ }^{1}$ Rajya Kappagantula, ${ }^{1}$ Alvin Makohon-Moore, ${ }^{5}$ Amber Johns, ${ }^{5}$ Antony Gill, ${ }^{1}$ Masataka Amisaki, 'Pablo Guasp, ${ }^{1}$ Abderezak Zebboudj, ${ }^{4}$ Rebecca Yu, ${ }^{4}$ Adrienne Kaya Chandra, ${ }^{4}$ Zagaa Odgerel, ${ }^{1}$ Michel Sadelain, ${ }^{1}$ Erin Patterson, ${ }^{1}$ Christine lacobuzio-Donahue, 'Benjamin Greenbaum, 'Vinod Balachandran. ${ }^{1}$ Memorial Sloan Kettering Cancer Center, New York, NY, United States; ${ }^{2}$ Tisch Cancer Institute at Mount Sinai, New York, NY United States; ${ }^{3}$ Memorial Sloan Cancer Center, New York, NY, United States; ${ }^{4}$ Memorial Sloan Kettering, New York, NY United States; ${ }^{5}$ The Kinghorn Cancer Centre, Darlinghurst, Australia

Background Cancer immunoediting predicts that $\mathrm{T}$ cells selectively kill tumor cells expressing immunogenic mutations (neoantigens) resulting in less immunogenic clones to outgrow in tumors. ${ }^{1}$ Although established through longitudinal studies of how tumors evolve in immune-proficient and -deficient mice, ${ }^{1}$ 2 whether the human immune system naturally targets neoantigens to edit tumors, and the principles that identify the edited neoantigens, remains unclear.

Methods To investigate if immune selective pressures on neoantigens alter how human tumors evolve, we longitudinally studied how 70 human pancreatic ductal adenocarcinomas (PDACs) - a poorly immunogenic cancer largely presumed to not be subject to immunoediting - evolved over 10 years. We use exome sequencing, neoantigen identification, and clonal reconstruction to compare how primary PDACs evolve to recurrence in rare long-term PDAC survivors previously shown to have more immunogenic tumors ${ }^{3}$ ( $\mathrm{n}=9$ patients, $\mathrm{n}=9$ primary, 22 recurrent tumors), to short-term survivors with less immunogenic primary tumors $(n=6$ patients, $n=6$ primary, 33 recurrent tumors). To identify immunogenic "high quality" neoantigens, we use neopeptide-T cell functional assays and computational modeling to extend and apply a previously developed neoantigen quality $\operatorname{model}^{3} 4$ by predicting high quality neoantigens as arising from amino acid substitutions with sufficient antigenic distance from cognate wild-type peptides to differentially bind the MHC or activate a $\mathrm{T}$ cell.

Results Compared to short-term survivors, we observe that long-term survivors evolve fewer recurrent tumors with longer latency, and distinct tissue tropism. To evaluate if differential immune pressures explained these differences, we discover that despite longer times to evolve, long-term survivors evolve genetically less heterogeneous tumors with fewer clones, fewer nonsynonymous mutations, and fewer neoantigens. To identify if high quality neoantigens are selectively edited in recurrent tumors of long-term survivors, we observe that neoantigens with greater antigenic distance ("less self") are more depleted in primary and recurrent tumors of long- compared to shortterm survivors. Furthermore, we find that long-term survivors evolve markedly fewer new neoantigens of strikingly lower quality, to indicate clones with high quality neoantigens are immunoedited.

Conclusions We submit longitudinal evidence that the human immune system naturally edits neoantigens in PDAC. Furthermore, we present a model that describes how cancer neoantigens evolve under immune pressure over time, with implications for cancer biology and therapy. More broadly, our results argue that immunoediting is a fundamental cancer suppressive mechanism that can be quantified to predict tumor evolution.

Acknowledgements This work was supported by NIH U01 CA224175 (V.P.B), a Stand Up to Cancer Convergence Award (B.D.G, V.P.B.), a Damon Runyon Clinical Investigator Award
(V.P.B), and the Avner Pancreatic Cancer Foundation (A.J, A. G). Services by the Integrated Genomics Core were funded by the NCI Cancer Center Support Grant (P30 CA08748), Cycle for Survival, and the Marie-Josée and Henry R. Kravis Center for Molecular Oncology.

\section{REFERENCES}

1. Shankaran $V$, et al. IFNgamma and lymphocytes prevent primary tumour development and shape tumour immunogenicity. Nature 2001;410:1107-1111.

2. Matsushita $\mathrm{H}$, et al. Cancer exome analysis reveals a T-cell-dependent mechanism of cancer immunoediting. Nature 2012;482:400-404.

3. Balachandran VP, et al. Identification of unique neoantigen qualities in long-term survivors of pancreatic cancer. Nature 2017:551:512-516.

4. Łuksza $M$, et al. A neoantigen fitness model predicts tumour response to checkpoint blockade immunotherapy. Nature 2017;551:517-520.

Ethics Approval This study was performed in strict compliance with all institutional ethical regulations and approved by the institutional review boards of Memorial Sloan Kettering Cancer Center (MSK), the Garvan Institute of Medical Research, and the The Johns Hopkins Hospital (JHH). We obtained informed consent from all patients.

http://dx.doi.org/10.1136/jitc-2021-SITC2021.824 